\title{
Notable roles of EZH2 and DNMT1 in epigenetic dormancy of the SHP1 gene during the progression of chronic myeloid leukaemia
}

\author{
JING WANG ${ }^{1}$, LUOMING HUA ${ }^{2}$, MING GUO $^{2}$, LIN YANG ${ }^{1}$, XIAOJUN LIU ${ }^{1}$, \\ YANMENG LI ${ }^{3}$, XIAOYAN SHANG ${ }^{3}$ and JIANMIN LUO ${ }^{1}$ \\ ${ }^{1}$ Department of Hematology, The Second Hospital of Hebei Medical University, Shijiazhuang, \\ Hebei 050017; ${ }^{2}$ Department of Hematology, Affiliated Hospital of Hebei University; \\ ${ }^{3}$ Clinical Medicine College of Hebei University, Baoding, Hebei 071000, P.R. China
}

Received January 29, 2016; Accepted December 20, 2016

DOI: $10.3892 / \mathrm{ol} .2017 .6050$

\begin{abstract}
Tumor development is associated with the methylation of cytosine-guanine $(\mathrm{CpG})$ islands. The occurrence of methylation requires several factors, such as DNA methylation systems and polycomb group (PcG) proteins. At present, novel drugs are needed for the treatment of chronic myeloid leukaemia (CML), particularly considering the current prognosis of CML. The methylation status of the Src homology 2 domain-containing tyrosine phosphatase 1 (SHP1) gene, a negative regulator of signal transduction, has been identified as being altered in numerous haematological malignancies. DNA methyltransferase 1 (DNMT1) and the PcG protein complex member enhancer of zeste homolog 2 (EZH2) participate in a number of gene methylation processes. The present study investigated the methylation status of the SHP1 gene in CML, and examined the association between DNMT1 and EZH2 activity and the SHP1 gene methylation status to develop novel strategies for the treatment of CML. The results revealed that SHP1 gene methylation status was altered during the progression of CML. These data indicated that SHP1 gene methylation is associated with the progression of this disease. The associations of DNMT1 and EZH2 activities with the methylation status of the SHP1 gene were additionally investigated via chromatin immunoprecipitation. DNMT1 and $\mathrm{EZH} 2$ were revealed to be bound to the promoter region of the SHP1 gene, and were involved in the process of SHP1 methylation. Furthermore, DNMT1 and EZH2 were associated with disease progression. Thus, the findings of the present study
\end{abstract}

Correspondence to: Dr Jianmin Luo, Department of Hematology, The Second Hospital of Hebei Medical University, 361 Zhongshan E Road, Shijiazhuang, Hebei 050017, P.R. China

E-mail: luojm2011@163.com

Key words: methylation, enhancer of zeste homolog 2, Src homology 2 domain-containing phosphatase 1, epigenetic dormancy, DNA methyltransferase 1, chronic myeloid leukaemia suggest a new target for the treatment of CML, particularly for future drug development.

\section{Introduction}

At present, tyrosine kinase inhibitors are often used for the treatment of chronic myeloid leukaemia (CML). However, certain patients with CML develop the acute phase of this disease due to drug resistance (1). To develop a rational treatment schedule and minimize drug resistance in the treatment of CML, additional investigation with respect to the pathogenic mechanism is required.

The tyrosine phosphatase SHP1 is a key negative regulator of intracellular signalling. Numerous studies have revealed that the expression of SHP1 is low in malignant lymphoma, acute leukaemia, multiple myeloma and other haematological malignancies, including myelodysplastic syndrome. However, the SHP1 gene was predominantly observed to be in a highly methylated state. Therefore, it was hypothesised that the methylation of the SHP1 gene serves an important role in the pathogenesis of malignant haematological diseases (2).

DNA methylation is an important epigenetic modification. DNA methyltransferases serve a role in gene methylation. At present, there are 3 known DNA methyltransferases (3): DNA methyltransferase 1 (DNMTl), DNMT2 and DNMT3a/b.

EZH2 is a member of the polycomb group $(\mathrm{PcG})$ gene family. Enhancer of zeste homolog 2 (EZH2) has been identified as key epigenetic regulator involved in transcriptional repression in haematological diseases (4). In mantle cell lymphoma, $\mathrm{EZH} 2$ has been identified to function in the regulation of homeobox (HOX) genes, and EZH2 activity may lead to high methylation levels of the HOX gene promoter region and extended gene silencing (5-7). This modification is associated with the onset of mantle cell lymphoma.

At present, there is limited research on the associations between DNMT1 and EZH2 activity and SHP1 methylation in CML. The present study investigated the association between these factors and SHP1 methylation via chromatin immunoprecipitation (ChIP), to identify the roles of DNMT1 and $\mathrm{EZH} 2$ in the process of blast crisis in CML. 


\section{Materials and methods}

Patients. A total of 60 patients with CML treated at the Affiliated Hospital of Hebei University (Baoding, China) from March 2014 to June 2015 were included in the present study. Among these patients, 35 were male and 25 were female, and the median age of the patients was 46 years with a range of 29-65 years. A total of 10 healthy donors were included as a control group. Subsequent to informed consent being obtained from all of the patients, mononuclear cells were collected by bone marrow aspiration. This study was approved by the ethics committee of the Affiliated Hospital of Hebei University (Baoding, China).

Cell culture and treatment. The CML cell line K562 (Beijing Institute for Cancer Research, Beijing, China) was maintained in the laboratory of the Department of Hematology of the Second Hospital of Hebei Medical University (Shijiazhuang, China). The cells were cultured in RPMI-1640 medium supplemented with $100 \mathrm{U} / \mathrm{ml}$ penicillin, $100 \mathrm{U} / \mathrm{ml}$ streptomycin and $10 \%$ foetal bovine serum. The cell lines were passaged every 2 to 3 days. The K562 cells were seeded at a density of $1 \times 10^{6}$ cells $/ \mathrm{ml}$ in $25 \mathrm{~cm}^{2}$ culture flasks, then treated with 5-aza-2'-deoxycytidine (decitabine; Sigma Aldrich; Merck KGaA, Darmstadt, Germany) or the inhibitor 3-deazaneplanocin A (DZNep; Sigma-Aldrich; Merck KGaA) Subsequently, the cells were harvested and used for western blot analysis, reverse transcription quantitative polymerase chain reaction (RT-qPCR), methylation-specific (MSP) PCR and ChIP.

Western blot analysis. The protein samples were separated on a $7.5-15 \%$ SDS-PAGE gel, and transferred to a polyvinylidene fluoride membrane. Subsequent to blocking of the non-specific binding sites with 5\% non-fat milk in TBS-Tween 20 (T; 20 mmol/l Tris-HCl, pH 7.4, 150 mmol/l $\mathrm{NaCl}$ and $0.1 \% \mathrm{~T}$ ) for $60 \mathrm{~min}$, the membranes were incubated overnight at $4^{\circ} \mathrm{C}$, with primary antibodies at 1:1,000 dilution. Anti-DNMT1 (\#ab13537) and anti-EZH2 (\#ab3748) antibodies were purchased from Abcam (Cambridge, UK) and an anti-SHP1 (\#sc52885) antibody was purchased from Santa Cruz Biotechnology, Inc., (Dallas, TX, USA). The membranes were then probed with a horseradish peroxidase-conjugated secondary antibody (\#sc2789; dilution, 1:5,000; Santa Cruz Biotechnology, Inc.) for $60 \mathrm{~min}$ at $37^{\circ} \mathrm{C}$. Subsequent to washing 3 times with TBS-T, the membranes were developed using an enhanced chemiluminescence detection system (LI-COR Biosciences, Lincoln, NE, USA).

$R T-q P C R$. The total RNA from the CML patient samples and K562 cell lines was extracted using TRIzol reagent (Invitrogen; Thermo Fisher Scientific, Inc.) according to the protocol of the manufacturer. Then, complementary (c) DNA was synthesized using an All-in-one ${ }^{\text {TII }}$ First-Strand cDNA Synthesis kit (GeneCopoeia Inc., Rockville, MD, USA) according to the manufacturer's protocol.

qPCR was then performed using an ABI7500 real-time PCR detection system (Applied Biosystems; Thermo Fisher Scientific, Inc. Waltham, MA, USA) with gene-specific primers. A $\beta$-actin cDNA fragment was used as an internal control. The sequences of all primers used in the RT-qPCR are provided in Table I. The PCR reaction contained $12 \mu \mathrm{l}$ of Platinum SYBR-Green qPCR SuperMix-UDG (Invitrogen; Thermo Fisher Scientific, Inc.), $1 \mu \mathrm{l}$ cDNA and $0.5 \mu \mathrm{l}$ of the forward and reverse primers. The PCR protocol was: $95^{\circ} \mathrm{C}$ for $2 \mathrm{~min}$, followed by 40 cycles of $95^{\circ} \mathrm{C}$ for $15 \mathrm{sec}, 60^{\circ} \mathrm{C}$ for $30 \mathrm{sec}$ and $68^{\circ} \mathrm{C}$ for $45 \mathrm{sec}$. The procedure was repeated 3 times and the $2^{-\Delta \Delta C q}$ method (8) was performed to quantify the gene expression level relative to the $\beta$-actin cDNA.

MSP. Genomic DNA from K562 cells and patients with CML isolated using the Rapid DNA Extraction kit (Bo Maide Biotechnology Co., Ltd., Beijing, China) was modified, via bisulphite treatment with a DNA methylation modification kit (Zymo Research Corp., Irvine, CA, USA). The genomic DNA was then amplified by PCR, using 2 sets of SHP1 promoter-specific primer pairs that recognized either a methylated or an unmethylated $\mathrm{CpG}$ sequence. The sequences of all primers used in the MSP PCR are provided in Table II. The PCR reaction mixes contained $2.5 \mu 1$ 10X Reaction Buffer, $1 \mu \mathrm{l}$ extracted DNA, $1 \mu \mathrm{l}$ of $5 \mu \mathrm{M}$ forward and reverse primers, $2 \mu 1$ of a $2.5 \mathrm{mM}$ dNTP mix, $2 \mu 1 \mathrm{MgCl}_{2}$ and $0.5 \mathrm{U}$ of DNA polymerase (Applied Biosystems; Thermo Fisher Scientific, Inc.). The thermocycler settings were as follows: $95^{\circ} \mathrm{C}$ for $10 \mathrm{~min}$, followed by 38 cycles of $95^{\circ} \mathrm{C}$ for $30 \mathrm{sec}$, $54^{\circ} \mathrm{C}$ for $30 \mathrm{sec}$ and $72^{\circ} \mathrm{C}$ for $30 \mathrm{sec}$. The reaction products were analysed by electrophoresis with an Invitrogen DNA ladder (Thermo Fisher Scientific, Inc.) and 10,000X GelRed ${ }^{\text {Tu }}$ Nucleic Acid Gel Stain (Biotium, Inc., Hayward, CA, USA).

ChIP. The ChIP assay was performed using a SimpleChIP ${ }^{\circledR}$ Enzymatic Chromatin Immunoprecipitation kit (Cell Signaling Technology, Inc., Danvers, MA, USA) and antibodies targeting DNMT1, EZH2 and rabbit IgG, as according to the manufacturer's protocol. The K562 cells were seeded at a density of $1 \times 10^{6}$ cells $/ \mathrm{ml}$ in $25 \mathrm{~cm}^{2}$ culture flasks, then incubated alone, with decitabine $(50 \mu \mathrm{M})$ or with DZNep $(10 \mu \mathrm{M})$ and grown to a final count of $5-10 \times 10^{7}$ cells for each ChIP experiment. Formaldehyde was added to the cells to a final concentration of $1 \%$ and the cells were re-suspended in SDS lysis buffer and immunoprecipitated. The resulting complexes were pelleted with protein A agarose and eluted with elution buffer containing $50 \mathrm{mM}$ Tris- $\mathrm{HCl}, 10 \mathrm{mM}$ EDTA and $1 \%$ SDS. Histone-DNA crosslinks were reversed in $5 \mathrm{M}$ sodium chloride, and DNA was recovered via phenol/chloroform extraction. The immunoprecipitated DNA and input samples were analysed by PCR. The following primers for the SHP1 gene promoter were used: Forward, 5'-ATGATAAAG ATAGCCCCTGTT-3'; reverse, 5'-TCATCGAGTGAGTCC TGCTG-3'. The PCR mixes contained $2 \mu \mathrm{l}$ DNA, $1 \mu 1$ of $5 \mu \mathrm{M}$ forward and reverse primers, $2 \mu \mathrm{l}$ of a $2.5 \mathrm{mM}$ dNTP mix, $2 \mu \mathrm{l}$ $\mathrm{MgCl}_{2}$ and $0.5 \mathrm{U}$ of DNA polymerase (Applied Biosystems; Thermo Fisher Scientific, Inc.). The thermocycler settings were as follows: $94^{\circ} \mathrm{C}$ for $10 \mathrm{~min}$, followed by 32 cycles of $94^{\circ} \mathrm{C}$ for $30 \mathrm{sec}, 59^{\circ} \mathrm{C}$ for $30 \mathrm{sec}$ and $72^{\circ} \mathrm{C}$ for $30 \mathrm{sec}$. The reaction products were then analyzed by electrophoresis as previously described.

Statistical analysis. The values were presented as the mean \pm standard deviation. Significant differences were assessed using SAS 9.1.3 statistical software (SAS Institute, 
Table I. Primers for reverse transcription quantitative polymerase chain reaction amplification.

\begin{tabular}{lc}
\hline Primers & Sequence \\
\hline SHP1 & \\
Sense & 5'-CACCATCATCCACCTCAA GT-3' \\
Antisense & 5'-TCTCAGCACAAGAA ACGTC-3' \\
DNMT1 & \\
Sense & 5'-TACCTGGACGACCCTGACCTC-3' \\
Antisense & 5'-CGTTGGCATCAAAGATGGACA-3' \\
EZH2 & \\
Sense & 5'-TTCATGCAACACCCA ACACT-3' \\
Antisense & 5'-GGGCCTGCTACTGTTATTGG-3' \\
$\beta$-actin & \\
Sense & 5'-TGACGTGGACATCCGCAAAG-3' \\
Antisense & 5'-CTGGAAGGTGGACAGCGAGG-3' \\
\hline
\end{tabular}

Inc., Cary, NC, USA). First, normality and variance homogeneity tests of the count data were performed. The data were then subjected to analysis of variance or the independent-samples t test. The clinical variables were analysed using the $\chi^{2}$ test and Fisher's exact test. $\mathrm{P}<0.05$ was considered to indicate a statistically significant difference.

\section{Results}

Expression of SHPl and the methylation of the promoter region of SHP1 in patients with CML. The present study detected the protein expression of SHP1 in the mononuclear cells from the bone marrow of patients with CML by western blot analysis. The results demonstrated that the protein expression of SHP1 increased in patients with chronic phase CML (CML-CP) and normal control (NC) subjects compared to patients with accelerated phase CML (CML-A) and patients with blast phase $\mathrm{CML}(\mathrm{CML}-\mathrm{BP} ; \mathrm{P}<0.05)$. There was no significant difference in the levels of SHP1 protein expression between NC subjects and patients with CML-CP ( $\mathrm{P}>0.05$ ) or between CML-AP and patients with CML-BP, as demonstrated in Fig. 1A. Methylation of SHP1 was detected in $7 / 33$ patients with CML-CP, positive rate of $21.2 \%$. However, methylation of the SHP1 gene was detected in all patients with advanced stage CML, including patients with CML-AP and CML-BP. The positive rate for SHP1 gene methylation in patients with advanced stage CML was significantly higher compared with patients with CML-CP $(\mathrm{P}<0.01)$. Specifically, 4 patients with CML-AP (40\%) and 8 patients with BP-CML (47\%) exhibited complete SHP1 promoter methylation, and this difference was not statistically significant $(\mathrm{P}>0.05)$, as illustrated in Fig. 1B.

High expression of EZH2 and DNMT1 in K562 cells and in patients with advanced CML. RT-qPCR analysis revealed that the relative mRNA levels of DNMT1 were 3.54 \pm 1.23 , $1.31 \pm 0.18,1.75 \pm 0.53,2.76 \pm 0.78$, and $3.05 \pm 1.23$ in the samples from K562 cells, NC donors, and patients with CML-CP, CML-AP and CML-BP, respectively. The mRNA levels of
Table II. Primers for methylation-specific polymerase chain reaction.

\begin{tabular}{llr}
\hline Primers & \multicolumn{1}{c}{ Sequence } & Length \\
\hline $\begin{array}{l}\text { M-MSP } \\
\text { Sense }\end{array}$ & $\begin{array}{l}\text { 5'-GAACGTTATTATAGTATA } \\
\text { GCGTTC-3' }\end{array}$ \\
Antisense & 5'-TCACGCATACGAACCCA & 159 bp \\
& AACG-3' & \\
U-MSP & & \\
Sense & 5'-GTGAATGTTATTATAGTAT & \\
& AGTGTTTGG-3' & \\
Antisense & 5'-TTCACACATACAAACCCA & 162 bp \\
& AACAAT-3' & \\
\hline
\end{tabular}

DNMT1 were significantly higher in the samples from K562 cells and patients with CML-AP and CML-BP than in those from NC subjects and patients with CML-CP $(\mathrm{P}<0.05)$. No significant difference in the DNMT1 mRNA levels between K562 cells and patients with CML-AP or CML-BP or between $\mathrm{NC}$ subjects and patients with CML-CP was observed ( $\mathrm{P}>0.05)$, as demonstrated in Table III.

RT-qPCR analysis demonstrated that the relative mRNA levels of EZH2 were $2.54 \pm 1.23,1.12 \pm 0.08,1.25 \pm 0.33$, $1.99 \pm 0.78$, and $2.15 \pm 1.01$ in the samples from the K562 cells, NC donors, and patients with CML-CP, CML-AP, and CML-BP, respectively. The mRNA levels of EZH2 were significantly higher in samples from K562 cells and patients with CML-AP and CML-BP compared with the samples from $\mathrm{NC}$ subjects and patients with CML-CP $(\mathrm{P}<0.05)$. No significant difference in the EZH2 mRNA levels between K562 cells and patients with CML-AP or CML-BP or between NC subjects and patients with CML-CP was observed $(\mathrm{P}>0.05)$, as demonstrated in Table III.

DNMT1 and SHPI expression prior and subsequent to treatment with decitabine. RT-qPCR analysis illustrated that the relative mRNA levels of DNMT1 and SHP1 in K562 cells were $3.54 \pm 1.23$ and $0.18 \pm 0.08$, respectively. Treatment with decitabine led to a decrease in the DNMT1 mRNA level to $1.55 \pm 0.61(\mathrm{P}<0.05)$, but significantly increased the SHP1 expression level to $1.08 \pm 0.20(\mathrm{P}<0.05)$, as demonstrated in Fig. 2A.

A similar pattern of the levels of SHP1 and DNMT1 expression was observed at the protein level, as demonstrated in Fig. 2B. In addition, subsequent to the application of decitabine, the methylation level of the SHP1 gene was decreased in the K562 cells, from complete methylation to partial methylation, as illustrated in Fig. $2 \mathrm{C}$.

EZH2 and SHP1 expression prior and subsequent to treatment with DZNep. RT-qPCR analysis demonstrated that the relative mRNA levels of EZH2 and SHP1 in K562 cells were $2.54 \pm 1.23$ and $0.18 \pm 0.08$, respectively. Treatment with DZNep led to a decrease in the EZH2 mRNA level to $1.80 \pm 0.62$ $(\mathrm{P}<0.05)$ but a significant increase in the relative mRNA level 
A

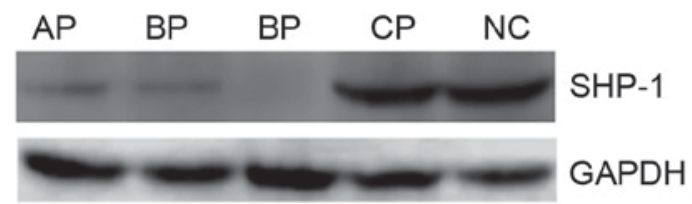

B

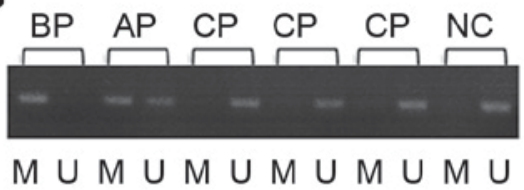

Figure 1. Expression of SHP1 protein and the methylation status of the SHP1 promoter in patients in different phases of chronic myelogenous leukaemia. (A) A western blot analysis to illustrate the relative protein expression level of SHP1. (B) Extent of CpG methylation of the SHP1 promoter, as determined with PCR using methylation-specific primers. U, unmethylated-specific primers; M, methylated-specific primers; AP, accelerated phase; BP, blast phase; CP, chronic phase; NC, normal control; CpG, Cytosine-Guanine sequence; SHP1, Src homology 2 domain-containing phosphatase-1.

Table III. Relative expression of DNMT1 and EZH2 in patients with CML and normal control.

\begin{tabular}{lccc}
\hline Groups & $\mathrm{n}$ & $\begin{array}{c}\text { Relative levels } \\
\text { of EZH2 RNA }\end{array}$ & $\begin{array}{c}\text { Relative levels } \\
\text { of DNMT1 RNA }\end{array}$ \\
\hline NC & 10 & $1.12 \pm 0.08$ & $1.31 \pm 0.18$ \\
CML-CP & 33 & $1.25 \pm 0.33$ & $1.75 \pm 0.53$ \\
CML-AP & 10 & $1.99 \pm 0.78$ & $2.76 \pm 0.78$ \\
CML-BP & 17 & $2.15 \pm 1.01$ & $3.05 \pm 1.23$ \\
\hline
\end{tabular}

$\mathrm{NC}$, negative control; CML, chronic myelogenous leukemia; $\mathrm{CP}$, chronic phase; AP, accelerated phase; BP, blast phase; EZH2 enhancer of zeste homolog 2; DNMT1, DNA methyltransferase 1.

of SHP1 to $0.75 \pm 0.18(\mathrm{P}<0.05)$, as illustrated in Fig. 3A. A similar pattern of SHP1 and EZH2 expression was observed at the protein level, as demonstrated in Fig. 3B. Furthermore, treatment with DZNep led to the progressive demethylation of SHP1, as illustrated in Fig. 3C. Overall, these data suggested that decitabine and DZNep successfully reduced the level of CpG hypermethylation, leading to a re-expression of SHP1 mRNA and protein.

DNMT1 bound to the SHP1 gene promoter region of K562 cells by ChIP. Subsequent to the addition of decitabine, the interaction between DNMT1 and the SHP1 promoter region was weakened. To demonstrate that DNMT1 is functionally involved in the methylation of the SHP1 gene promoter and the inhibition of SHP1 gene expression, ChIP assays using anti-DNMT1 antibodies were performed, and DNMT1 was revealed to be enriched in the SHP1 promoter region of the K562 cells. Next, drug intervention experiments on K562 cells using the DNA methyltransferase inhibitor decitabine were carried out. The expression of SHP1 increased in the K562 cell line subsequent to decitabine treatment. Under these conditions, the interaction between DNMT1 and the SHP1 gene promoter region decreased based on ChIP, as illustrated in Fig. 2D. The methylation of the SHP1 promoter region also decreased subsequent to decitabine treatment.

EZH2 bound to the SHP1 gene promoter region in K562 cells by CHIP. Subsequent to the addition of DZNep, the interaction between EZH2 and the SHP1 promoter region was weakened. To identify the epigenetic control of SHP1 in K562 cells, the role of EZH2 in the regulation of SHP1 expression was investigated. To evaluate DNA methylation, ChIP assays were performed. In K562 cells, the SHP1 core region displayed enriched binding to EZH2. To confirm that EZH2 regulates SHP1 expression, K562 cells were treated with DZNep. Treatment with DZNep was revealed to increase SHP1 expression, and the results of the ChIP assay demonstrated reduced binding efficiency of EZH2 to the SHP1 promoter in the DZNep-treated cells, as illustrated in Fig. 3D. In addition, DZNep treatment led to decreased SHP1 methylation.

\section{Discussion}

CML is one of the most common types of malignant haematological tumor. At present, imatinib is the first-line drug for the treatment of CML. However, certain patients cannot afford long-term imatinib therapy or are resistant to second-generation kinase inhibitors. Therefore, it is necessary to explore novel treatment approaches from the perspective of CML pathogenesis.

Methylation in the promoter regions of tumor suppressor genes leads to tumor formation $(9,10)$. The tyrosine phosphatase SHP1 is a key negative regulator of intracellular signalling and is hypothesised to be a tumor suppressor gene. The methylation status of the SHP1 promoter is associated with gene dormancy, which is an important mechanism in the development of lymphoma and leukaemia. SHP1 protein expression was completely absent or partially reduced in the majority of types of lymphoma and leukaemia cell lines, such as YS2, IWA3, NK, TomJim, MTI, EDS, and ATLIK $(11,12)$. The present study demonstrated the lack of SHP1 expression in K562 cells. However, the SHP1 gene promoter was highly methylated. The degree of SHP1 promoter methylation increased with decreasing SHP1 expression in patients with CML-BP. In contrast, the degree of SHP1 promoter methylation decreased with increasing SHP1 expression in patients with CML-CP. These data suggested that the SHP1 gene methylation status in patients with CML-BP may be associated with the disease progression of CML.

Decitabine, a methyltransferase inhibitor, has been applied to other haematological diseases, such as myelodysplastic syndromes (MDS) and acute myelogenous leukaemia (AML). Therefore, the present study investigated the activity of methyltransferases in CML. The expression of DNMT1 was revealed to be significantly increased in patients with CML-BP and in K562 cells. The expression of DNMT1 decreased subsequent to the application of decitabine. Additionally, 
$\mathbf{A}$

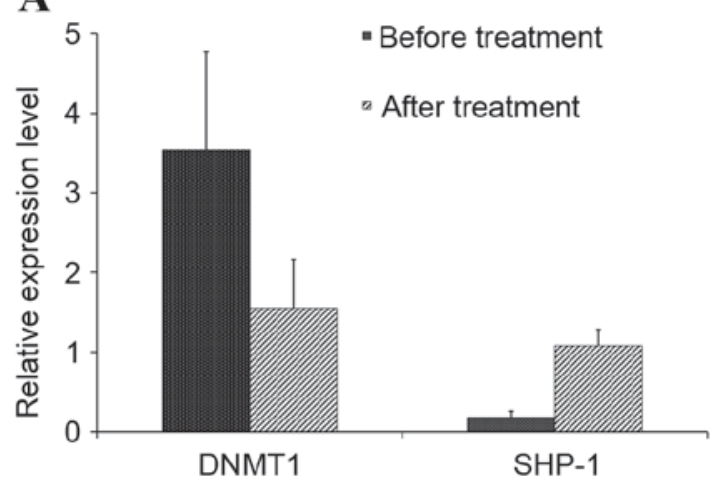

B K562 5-aza-CdR

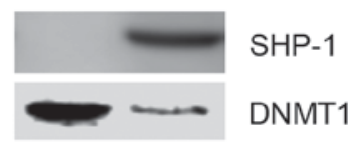

C

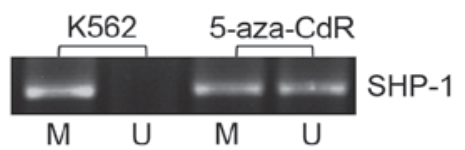

D $\quad \begin{array}{llllllll}M & 1 & 2 & 3 & 4\end{array}$

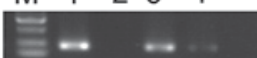

Figure 2. Effect of decitabine on K562 cells. (A) Relative expression level of DNMT1 and SHP1 mRNA. Error bars represent standard deviation. (B) A western blot to demonstrate the relative expression of SHP1 and DNMT1 proteins. (C) Extent of CpG methylation of the SHP1 promoter, as determined with PCR using methylation-specific primers. (D) The extent of DNMT1 binding to the SHP-1 promoter. Cell lysates were examined in the chromatin immunoprecipitation assay. U, unmethylated-specific primers; (C) M, methylated-specific primers; (D) M, marker; 1, input; 2, negative control; 3, prior to treatment with decitabine; 4, subsequent to treatment with 5-aza-2'-deoxycytidine (decitabine); decitabine, 5-aza-2'-deoxycytidine; SHP1, Src homology 2 domain-containing phosphatase-1; DNMT1, DNA methyltransferase 1.

A

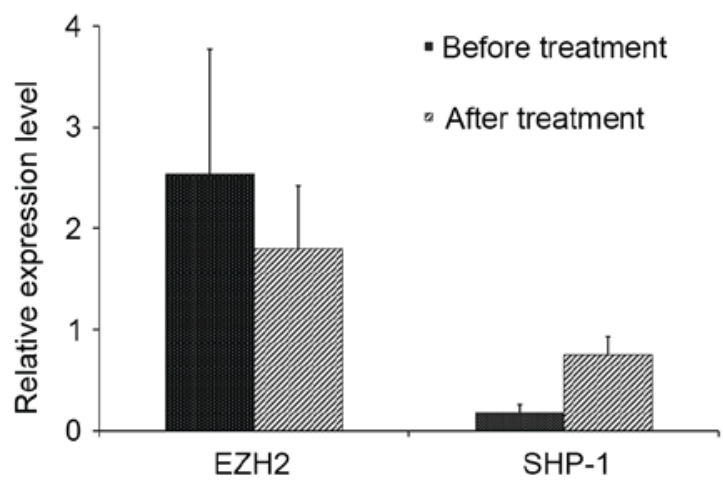

B
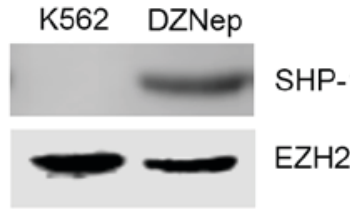

C

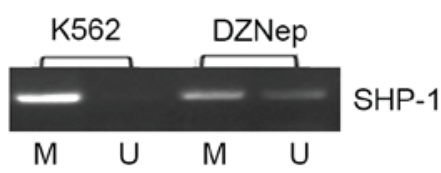

D

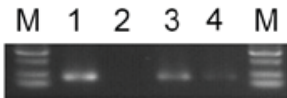

Figure 3. Effect of DZNep on K562 cells. (A) Relative expression level of EZH2 and SHP1 mRNA. Error bars represent standard deviation. (B) A western blot to demonstrate the relative expression of SHP1 and EZH2 proteins. (C) Extent of CpG methylation of the SHP1 promoter, as determined with PCR using methylation-specific primers. (D) The extent of EZH2 binding to the SHP-1 promoter. Cell lysates were examined with a chromatin immunoprecipitation assay. U, unmethylated-specific primers; (C) M, methylated-specific primers; (D) M, marker; 1, input; 2, negative control; 3, prior to treatment with DZNep; 4, subsequent to treatment with DZNep. DZNep, 3-deazaneplanocin A; SHP1, Src homology 2 domain-containing phosphatase-1; EZH2, enhancer of zeste homolog 2 .

the degree of SHP1 promoter methylation decreased with increasing SHP1 expression. Thus, it was hypothesised that DNMT1 may be associated with SHP1 gene methylation and CML disease progression. In the present study, based on ChIP assays, it was revealed that DNMT1 binds to the SHP1 promoter region under control conditions and that the interaction between DNMT1 and the SHP1 gene promoter region and the methylation of SHP1 significantly decreased following inhibitor treatment. These results indicated that a methyltransferase interacts with the SHP1 gene promoter and is involved in SHP1 gene methylation, thus affecting the blast crisis of CML. The present study established a theoretical basis for the treatment of CML with methyltransferase inhibitors.

In determining whether only the DNMT family participates in the methylation of SHP1, it was demonstrated that the PcG protein was one of the most important types of epigenetic regulators involved in the inhibition of gene activity. To support the future development of additional therapeutic targets for the treatment of CML, EZH2 was investigated.
EZH2 is a member of the PcG histone methyltransferase complex, which is a group of proteins associated with histone methyltransferases that are involved in the inhibition of transcription. The overexpression of the EZH2 gene is associated with the progression of malignant tumors and poor prognosis (13-15).

The EZH2 gene expression level in patients with high-risk MDS or AML was significantly higher compared with patients with low-risk MDS and in normal subjects (4). These results suggested that EZH2 serves a role in the development of myeloid malignancies. Similar to the observations in other types of malignancy, in mantle cell lymphoma the chromatin modifier EZH2 is overexpressed in proliferating cells and is associated with poor outcome (15-17).

EZH2 catalyses the trimethylation at lysine 27 of histone $\mathrm{H} 3$ (H3K27me3) (18), which serves as an anchorage point for the recruitment of additional PcG proteins and contributes to the formation of a repressive chromatin state. Consequently, EZH2 may indirectly inhibit target gene expression. The 
majority of these target genes inhibit tumor formation and regulate stem cell differentiation. Therefore, silencing these genes may lead to tumor formation. Inhibiting EZH2 activity in mouse models has been demonstrated to completely inhibit tumor growth (19). According to the transcriptional inhibition of PcG, there are 1,000 silencing target genes in human embryonic fibroblasts, which contribute to embryonic development, including members of the Notch, Hox, Hedgehog, Wnt, transforming growth factor (TGF) and fibroblast growth factor (FGF) signalling pathways $(17,20)$.

In solid tumors, there were a number of studies concerning $\mathrm{EZH} 2$. For example, it was reported that MYC and EZH2 function as potent suppressors of levels of macrophage stimulating 1 (MST1) expression in human prostate cancer cells Pharmacological and RNAi experiments revealed that MYC and EZH2 inhibit the promoter activity of MST1 and thus silence expression, and that EZH2 is a mediator of the MYC-induced silencing of MST1 (21).

EZH2 has been reported to directly control DNA methylation by regulating the activity of DNA methyltransferases in haematological malignancies. A ChIP study demonstrated that DNMT1, DNMT3a, DNMT3b and EZH2 bound to the promoter region of MYT1, and that the number of these interactions correlated with the methylation status of MYT1. Similar experiments confirmed that WNT1, KCNA1, and CNR1 were targeted by EZH2 (22).

In addition, it was revealed that EZH2 and DNMT3a localized to the PTEN promoter region in the EOL-1 cell line, which is resistant to imatinib, thus, participating in PTEN methylation. PTEN acts as a negative regulatory gene, as decreasing PTEN expression may cause cells to become drug-resistant (23). Additionally, in acute lymphoblastic leukaemia, EZH2 and $\mathrm{H} 3 \mathrm{~K} 27 \mathrm{me} 3$ were revealed to interact with the PTEN promoter region, and these interactions are associated with PTEN gene methylation. In summary, these data indicate that the PcG family protein EZH2 may be involved in CML pathogenesis by inhibiting the expression of the tumor suppressor gene PTEN (24). Therefore, in haematological diseases, the PTEN, WTY1 and HOX genes may be targets of EZH2. Gene silencing induced by DNA methylation requires EZH2 and DNA methylation enzymes $(22,25)$.

At present, a small number of studies have focused on the association between EZH2 and SHP1 gene methylation. Previous studies have demonstrated that SHP1 is highly methylated in diffuse large B cell lymphoma (DLBCL) $(26,27)$. The role of histone modifications was investigated via ChIP assays, which demonstrated that the P2 region of SHP1 was associated with the silencing of the histone mark H3K27me3 in DLBCL cells. Treatment with DZNep, an inhibitor of $\mathrm{EZH} 2$, decreased the levels of the $\mathrm{H} 3 \mathrm{~K} 27 \mathrm{me} 3$ mark within the P2 region of SHP1, resulting in re-expression of SHP1. The previous studies revealed novel epigenetic mechanisms of SHP1 suppression in DLBCL. However, additional studies are required to establish the effect of histone modifications, particularly the EZH2-mediated histone modifications on SHP1 in CML.

Based on the aforementioned previous data, including the high methylation of the SHP1 gene in patients with CML, it was hypothesised that EZH2 also participates in the process of SHP1 gene methylation. In the present study, a high level of methylation of the SHP1 gene in patients with CML-BP was detected. The methylation status of SHP1 decreased subsequent to the application of an inhibitor of EZH2. Therefore, assays were performed on K562 cells prior and subsequent to treatment with an inhibitor of EZH2. The SHP1 gene promoter was revealed to enrich binding to $\mathrm{EZH} 2$. Subsequent to drug intervention, the interaction of EZH2 with the promoter of SHP1 weakened, and the methylation status of SHP1 reduced. Therefore, it was hypothesised that EZH2 and DNMT1 are involved in SHP1 gene methylation, which may be associated with the progression of CML.

The present study demonstrates that EZH2 and DNMT1 interact with the SHP1 gene promoter and these interactions are associated with the SHP1 gene methylation status, which may lead to disease progression. The results of the present study may provide novel therapeutic targets for CML treatment in the future.

\section{Acknowledgements}

The present study would sincerely like to thank Ms. Yan Qin and Mr. Zheng LS for technical assistance, and Ms. Shi JH for valuable discussion in the course of the study.

\section{References}

1. Cools J, Maertens C and Marynen P: Resistance to tyrosine kinase inhibitors: Calling on extra forces. Drug Resist Updat 8: 119-129, 2005.

2. Sato H, Oka T, Shinnou Y, Kondo T, Washio K, Takano M, Takata K, Morito T, Huang X, Tamura M, et al: Multi-step aberrant $\mathrm{CpG}$ island hyper-methylation is associated with the progression of adult T-cell Leukemia/lymphoma. Am J Pathol 176: 402-415, 2010.

3. Cheng X and Blumenthal RM: Mammalian DNA methyltransferases: A structural perspective. Structure 16: 341-350, 2008.

4. Xu F, Li X, Wu L, Zhang Q, Yang R, Yang Y, Zhang Z, He Q and Chang C: Overexpression of the EZH2, RINGl and BMIl genes is common in myelodysplastic syndromes: Relation to adverse epigenetic alteration and poor prognostic scoring. Ann Hematol 90: 643-653, 2011.

5. Nagel S, Venturini L, Marquez VE, Meyer C, Kaufmann M, Scherr M, MacLeod RA and Drexler HG: Polycomb repressor complex 2 regulates HOXA9 and HOXA10, activating ID2 in NK/T-cell lines. Mol Cancer 9: 151, 2010.

6. Wu X, Gong Y, Yue J, Qiang B, Yuan J and Peng X: Cooperation between EZH2, NSPc1-mediated histone H2A ubiquitination and Dnmt1 in HOX gene silencing. Nucleic Acids Res 36: 3590-3599, 2008 .

7. Kanduri M, Sander B, Ntoufa S, Papakonstantinou N, Sutton LA, Stamatopoulos K, Kanduri C and Rosenquist R: A key role for EZH2 in epigenetic silencing of HOX genes in mantle cell lymphoma. Epigenetics 8: 1280-1288, 2013.

8. Livak KJ and Schmittgen TD: Analysis of relative gene expression data using real-time quantitative PCR and the 2(-Delta Delta C(T)) method. Methods 25: 402-408, 2001.

9. Qi J, Zhu YQ, Luo J, Tao WH and Zhang JM: Hypermethylation and regulation of expression of secreted frizzled-related protein genes in colorectal tumor. Zhonghua Zhong Liu Za Zhi 29: 842-845, 2007 (In Chinese).

10. Reddy J, Shivapurkar N, Takahashi T, Parikh G, Stastny V, Echebiri C, Crumrine K, Zöchbauer-Müller S, Drach J, Zheng Y, et al: Differential methylation of genes that regulate cytokine signaling in lymphoid and hematopoietic tumors. Oncogene 24: 732-736, 2005.

11. Migone TS, Cacalano NA, Taylor N, Yi T, Waldmann TA and Johnston JA: Recruitment of SH2 containing protein tyrosinephosphatase SHP-1 to the interleukin 2 receptor; loss of SHP-1 expression in human T-lymphotropic virus type I transformed T cells. Proc Natl Acad Sci USA 95: 3845-3850, 1998. 
12. Uhm KO, Lee ES, Lee YM, Park JS, Kim SJ, Kim BS, Kim HS and Park SH: Differential methylation pattern of ID4, SFRP1, and SHP1 between acute myeloid leukemia and chronic myeloid leukemia. J Korean Med Sci 24: 493-497, 2009.

13. Chase A and Cross NC: Aberrations of EZH2 in cancer. Clin Cancer Res 17: 2613-2618, 2011.

14. He IJ, Cai MY, Xu GL, Li JJ, Weng ZJ, Xu DZ, Luo GY, Zhu SL and Xie D: Prognostic significance of overexpression of EZH2 and $\mathrm{H} 3 \mathrm{k} 27 \mathrm{me} 3$ proteins in gastric cancer. Asian Pac J Cancer Prev 13: 3173-3178, 2012.

15. McCabe MT, Ott HM, Ganji G, Korenchuk S, Thompson C, Van Aller GS, Liu Y, Graves AP, Della Pietra A III, Diaz E, et al: EZH2 inhibition as a therapeutic strategy for lymphoma with EZH2-activating mutations. Nature 492: 108-112, 2012.

16. Fiskus W, Wang Y, Sreekumar A, Buckley KM, Shi H, Jillella A, Ustun C, Rao R, Fernandez P, Chen J, et al: Combined epigenetic therapy with the histone methyltransferase EZH2 inhibitor 3-deazaneplanocin A and the histone deacetylase inhibitor panobinostat against human AML cells. Blood 114: 2733-2743, 2009.

17. Simon JA and Lange CA: Roles of the EZH2 histone methyltransferase in cancer epigenetics. Mutat Res 647: 21-29, 2008.

18. Cao R, Tsukada Y and Zhang Y: Role of Bmi-1 and Ring1A in H2A ubiquitylation and Hox gene silencing. Mol Cell 20: $845-854,2005$

19. Wilson BG, Wang X, Shen X, McKenna ES, Lemieux ME, Cho YJ, Koellhoffer EC, Pomeroy SL, Orkin SH and Roberts CW: Epigenetic antagonism between polycomb and SWI/SNF complexes during oncogenic transformation. Cancer Cell 18: 316-328, 2010.
20. Bracken AP, Dietrich N, Pasini D, Hansen KH and Helin K: Genome-wide mapping of polycomb target genes unravels their roles in cell fate transitions. Genes Dev 20: 1123-1136, 2006.

21. Kuser-Abali G, Alptekin A and Cinar B: Overexpression of MYC and EZH2 cooperates to epigenetically silence MST1 expression. Epigenetics 9: 634-643, 2014.

22. Vire E, Brenner C, Deplus R, Blanchon L, Fraga M, Didelot C, Morey L, Van Eynde A, Bernard D, Vanderwinden JM, et al: The polycomb group protein EZH2 directly controls DNA methylation. Nature 439: 871-874, 2006.

23. Nishioka C, Ikezoe T, Yang J, Udaka K and Yokoyama A: Imatinib causes epigenetic alterations of PTEN gene via upregulation of DNA methyltransferases and polycomb group proteins. Blood Cancer J 1: e48, 2011.

24. Chen JH: Epigenetic studies of mesenchymal and B-cell leukemia. Beijing: Chinese Academy of Medical Sciences, Peking Union Medical College, 2010.

25. Sharma A, Heuck CJ, Fazzari MJ, Mehta J, Singhal S, Greally JM and Verma A: DNA methylation alterations in multiple myeloma as a model for epigenetic changes in cancer. Wiley Interdiscip Rev Syst Biol Med 2: 654-669, 2010.

26. Witzig TE, Hu G, Offer SM, Wellik LE, Han JJ, Stenson MJ, Dogan A, Diasio RB and Gupta M: Epigenetic mechanisms of protein tyrosine phosphatase 6 suppression in diffuse large B-cell lymphoma: Implications for epigenetic therapy. Leukemia 28: $147-154,2014$.

27. Chen X, Fu R, Wang Y, Song W, Ruan E, Qu W, Wang H, Wang G, Song J, Wang X, et al: Plasma DNA methylation of shp1 in patients with diffuse large B cell lymphoma. Zhonghua Yi Xue Za Zhi 94: 1071-1075, 2014 (In Chinese). 